in vivo $34: 2153-2157(2020)$

doi:10.21873/invivo.12022

\title{
Vietnamese Case Series of Hirayama Disease
}

\author{
NGUYEN DUY HUNG ${ }^{1,2 *}$, NGUYEN MINH DUC $34^{3 *}$, NGUYEN THANH VAN ${ }^{2}$, \\ LE THANH DUNG ${ }^{2}$, HOANG DUC HA ${ }^{5}$ and NGUYEN DUY HUE ${ }^{1,2}$ \\ ${ }^{1}$ Department of Radiology, Hanoi Medical University, Hanoi, Vietnam; \\ ${ }^{2}$ Department of Radiology, Viet Duc Hospital, Hanoi, Vietnam; \\ ${ }^{3}$ Department of Radiology, Pham Ngoc Thach University of Medicine, Ho Chi Minh City, Vietnam; \\ ${ }^{4}$ Department of Radiology, Children's Hospital 2, Ho Chi Minh City, Vietnam; \\ ${ }^{5}$ Department of Radiology, Haiphong University of Medicine and Pharmacy, Hai Phong City, Vietnam
}

\begin{abstract}
Background/Aim: Hirayama disease, a rare neurological disorder, is characterized by progressive, asymmetric muscle atrophy of the forearm and hand in young male patients. Case Report: We noted two cases of Hirayama disease that appeared in the right upper limb and identified the cervical neutral and flexion position magnetic resonance imaging (MRI) characteristics. Conclusion: In the flexion posture, the cervical MRI allows early diagnosis of Hirayama disease and should be considered in patients with the typical clinical symptoms.
\end{abstract}

Hirayama's disorder is characterized by the distal upper limbs' progressive, asymmetric muscle atrophy. This can be unilateral or bilateral and appear in the absence of pyramidal and tactile disorders (1). The disease usually occurs in young men aged 15 to 17 years in Asian countries such as Japan, Singapore and India (2). Muscle impairment and atrophy occur predominantly in the forearm and hand. The brachioradialis is denervated but the root of the C6 nerve is usually retained. The persistent denervation of the $\mathrm{C} 7$ to $\mathrm{T} 1$ myotomes is mainly observed by electromyograms (3). This disease's pathophysiological mechanism is documented as chronic ischemia in the anterior horns of the lower cervical spinal cord due to compression of the vertebral bone, particularly in the flexion position (4). Here, we present two cases of Hirayama disease occurring in the upper right limb

This article is freely accessible online.

*These Authors contributed equally to this study.

Correspondence to: Nguyen Minh Duc, MD, Department of Radiology, Pham Ngoc Thach University of Medicine, Ho Chi Minh City, Vietnam. E-mail: bsnguyenminhduc@pnt.edu.vn ORCID: 0000-0001-5411-1492

Key Words: Hirayama disease, cervical MRI, neutral position, flexion position. and describe the characteristics of the cervical magnetic resonance imaging (MRI) in neutral and flexion positions. The institutional review board of Viet Duc hospital approved this study. Informed consent was obtained from both patients.

\section{Case Series}

Case 1. A 16-year-old male with right-hand and forearm muscle weakness and atrophy slowly progressing for 3 years. Neurological analysis revealed the atrophy of the dorsal interosseous muscles, the thenar, hypothenar and forearm muscles with brachioradialis preservation (Figure 1). No visual impairment and pyramid syndrome were found.

Electromyogram showed the chronic denervation of the right first dorsal interosseous muscle and the right opponens pollicis muscle. The right brachioradialis was spared. The cervical spinal X-ray was normal.

The cervical MRI in neutral position revealed a focal atrophy of the spinal cord at C5-C6 levels with no signal abnormality on sagittal $\mathrm{T} 2$-weighted images and a flattening of the cord on axial T2-weighted images. No compression factor was detected (Figure 2). Since the image characteristics and clinical symptoms strongly suggested Hirayama disease, cervical MRI in flexion position was indicated. The posterior dural wall on flexion MRI was anteriorly displaced, causing narrowing of the posterior epidural area and spinal stenosis. The spinal cord at stages C5-C6 was anteriorly squeezed between the dura and the vertebral body (Figure 3). Based on the clinical results and characteristics of the flexion cervical MRI the patient was diagnosed with Hirayama disease.

Case 2. An 18-year-old male sought medical diagnosis due to right-hand deficiency, which had existed for 2 years and escalated dramatically in the last 3 months. Clinical examination revealed the wasting of the dorsal interosseous muscle and the palmar aspect muscles, as well as weakness in 


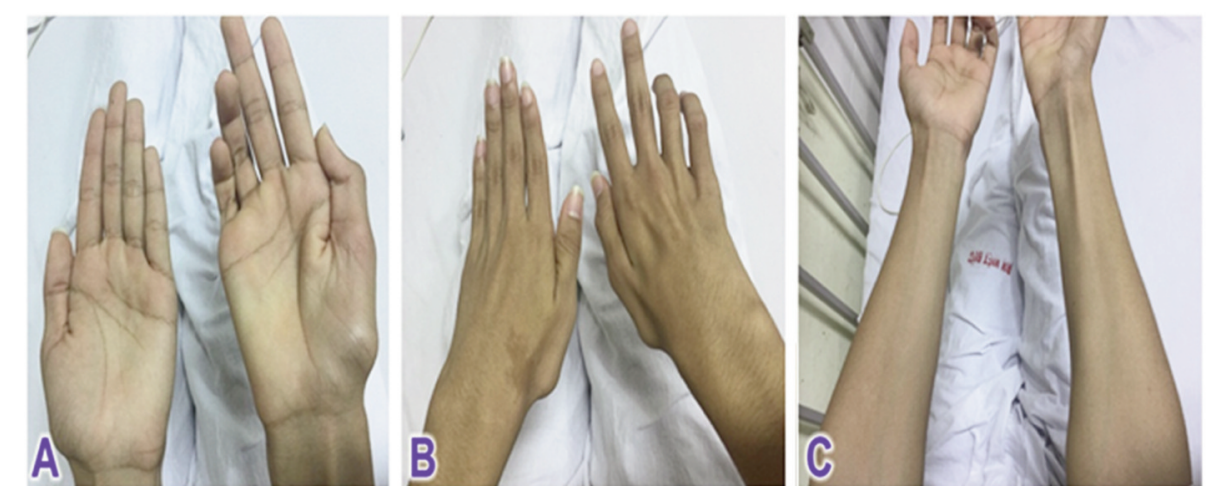

Figure 1. A 16-year-old male clinically suggested to have Hirayama disease. (A) and (B) The atrophy of dorsal interosseous muscles of the hand, the thenar, hypothenar muscles. (C) The atrophy of the medial aspect of forearm muscles with preservation of the brachioradialis.

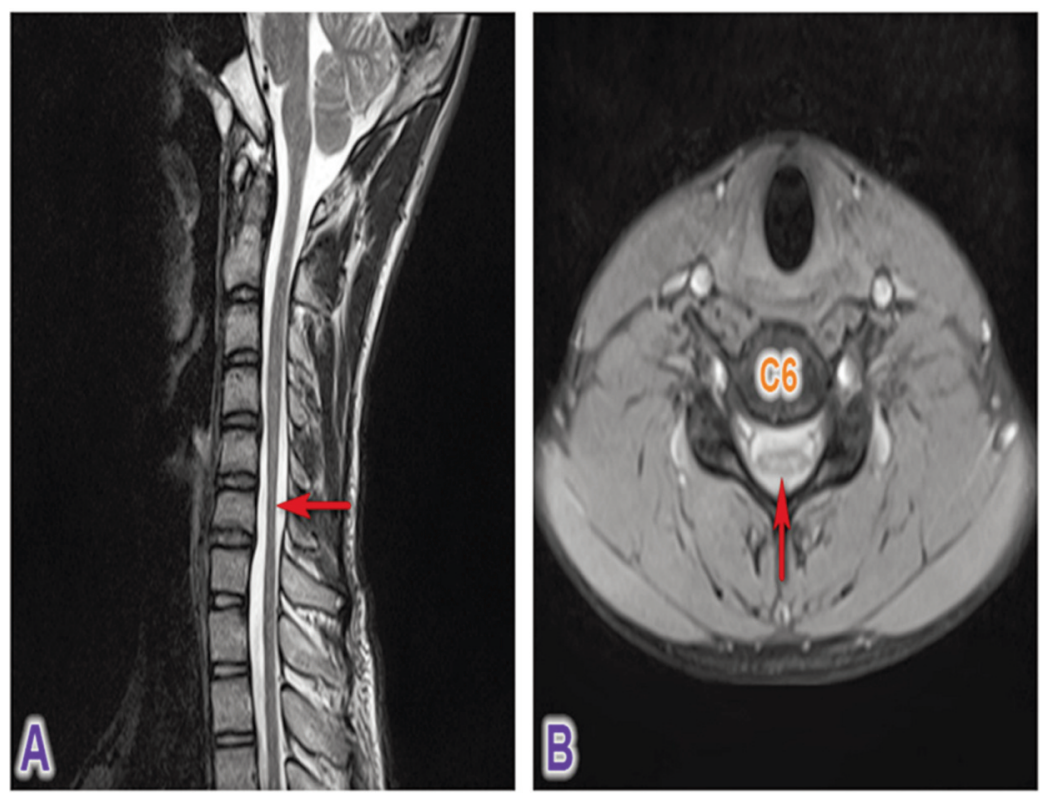

Figure 2. Cervical MRI of the 16-year-old patient in the neutral position. (A) Sagittal T2-weighted image showing a focal atrophy of the spinal cord at C5-C6 levels with no signal abnormality (arrow) (B) Axial T2-weighted image at C6 level showing flattening of the cord (arrow).

finger extension. The depletion of the medial aspect of forearm muscles with brachioradialis sparing was also noted (Figure 4). Pyramidal syndromes and sensory deficits were absent.

The electromyogram revealed signs of mere neurogenic motor injury that affected the right distal upper limb without any sensory impairment. No abnormality on cervical X-ray was observed. The neutral cervical MRI revealed a subtle medullary atrophy on sagittal T2-weighted image at C5-C6 stages and on axial T2-weighted image, the chord was stretched and the posterior dura somewhat was separated from the bone (Figure 5). The medullary signal was normal. No compression element was observed. Hirayama disease was presumed according to MRI attributes and clinical symptoms. Thus, the patient underwent the cervical MRI in flexion position. On sagittal and axial T2-weighted images, enlargement of the posterior epidural space was clearly detected due to the anterior displacement of the posterior dural wall causing the spinal stenosis (Figure 6).

\section{Discussion}

Hirayama disease, also known as "juvenile muscular atrophy of unilateral upper extremity", is a rare neurological disease that was first described in 1959 by Hirayama et al (5). It is more commonly observed in young men aged 15 to 17 years $(89.2 \%)$ (3). The lesions usually occur in the anterior horns 


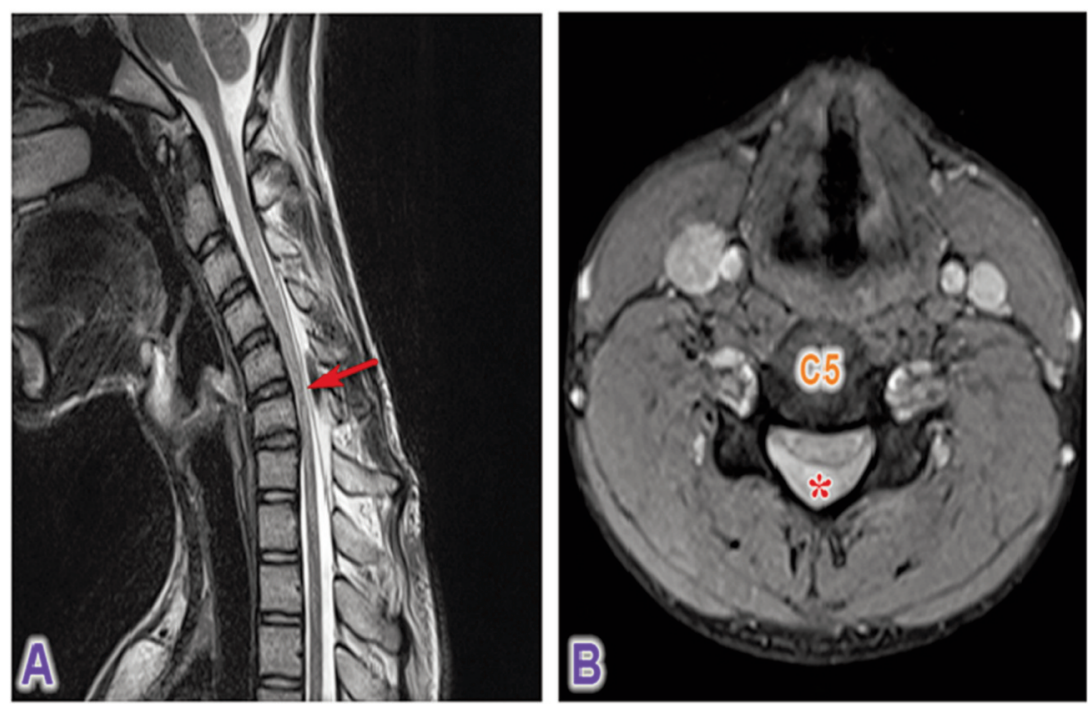

Figure 3. Cervical MRI of the 16-year-old patient in the flexion position. (A) Sagittal T2-weighted and (B) Axial T2-weighted images at C5 level showing the posterior dura was displaced anteriorly (arrow) causing the widening of posterior epidural space (asterisk) and spinal stenosis. The spinal cord at C5-C6 levels was compressed between the dura posteriorly and the vertebrae anteriorly.
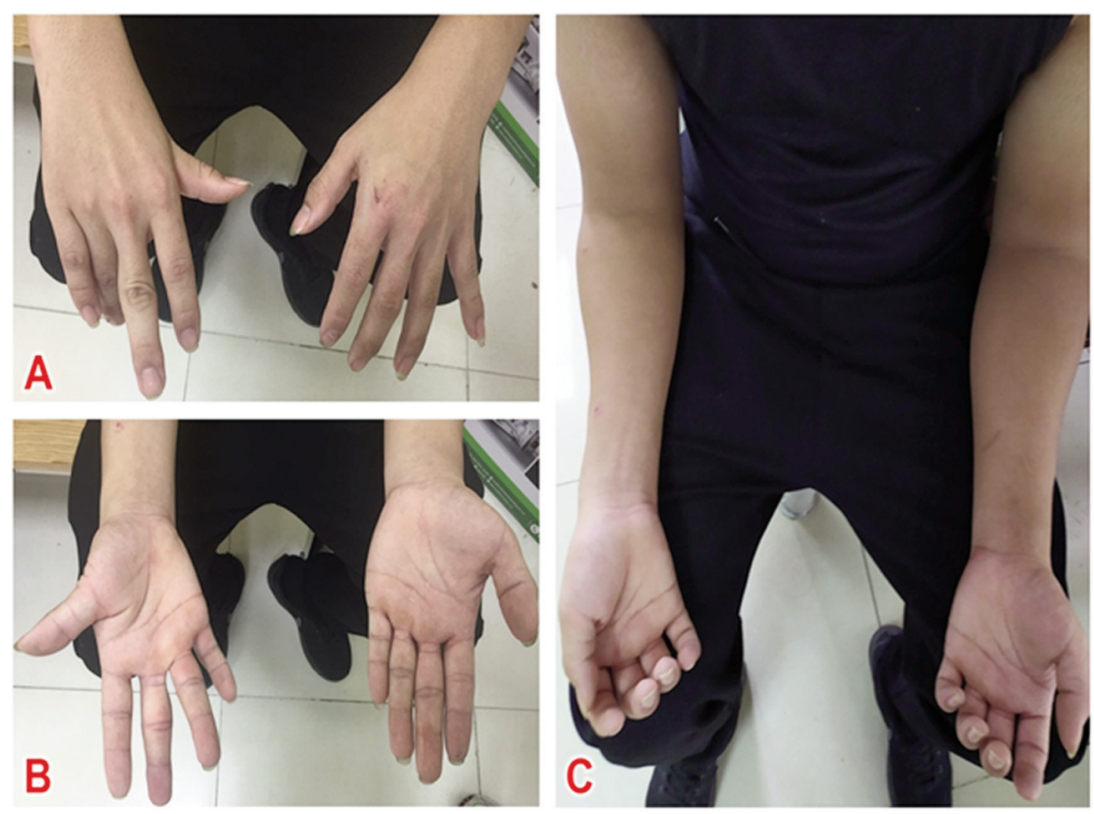

Figure 4. An 18-year-old male clinically suggested to have Hirayama disease. (A) and (B) The wasting of the dorsal interosseous muscle and the palmar aspect muscles, as well as weakness in finger extension. (C) The wasting of the medial aspect of forearm muscles with preservation of the brachioradialis.

of the lower cervical and upper thoracic spinal cord, particularly at levels $\mathrm{C} 7, \mathrm{C} 8$ and $\mathrm{T} 1$, triggering unilateral, asymmetric motor deficits that are exacerbated by neck flexion. The disorder occurs in two phases with symptoms deteriorating in the first 1-3 years, followed by a stabilization after about 5 years $(2,6)$. The indicative clinical signs are unilateral of right-side preponderance, asymmetric atrophy and weakness of the hand and the anterior aspect of forearm muscles (7). Muscle weakness in the hands exists in those with ulnar nerve denervation and rarely in those with median nerve denervation. In the forearm, the occurrence of "oblique atrophy" with brachioradialis conservation is the 


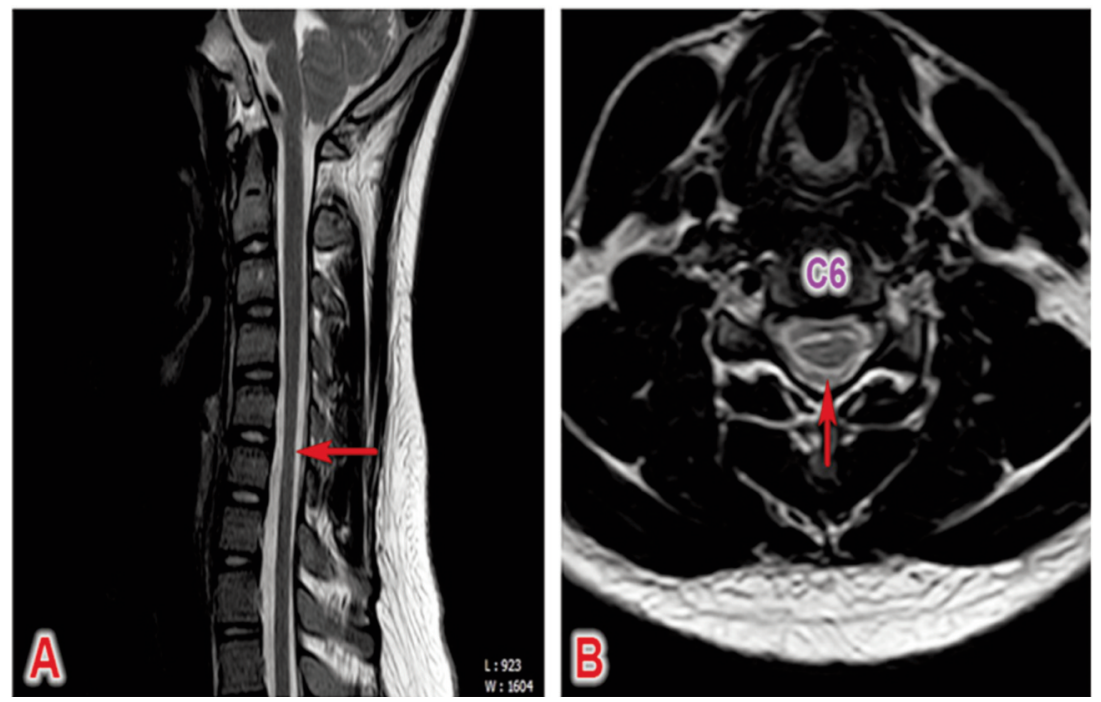

Figure 5. Cervical MRI of the 18-year-old patient in the neutral position. (A) Sagittal T2-weighted image showing a subtle medullary atrophy at C5-C6 levels with no signal abnormality (arrow) (B) Axial T2-weighted image at C6 level showing a flattening of the cord and detachment of the posterior dura (arrow).
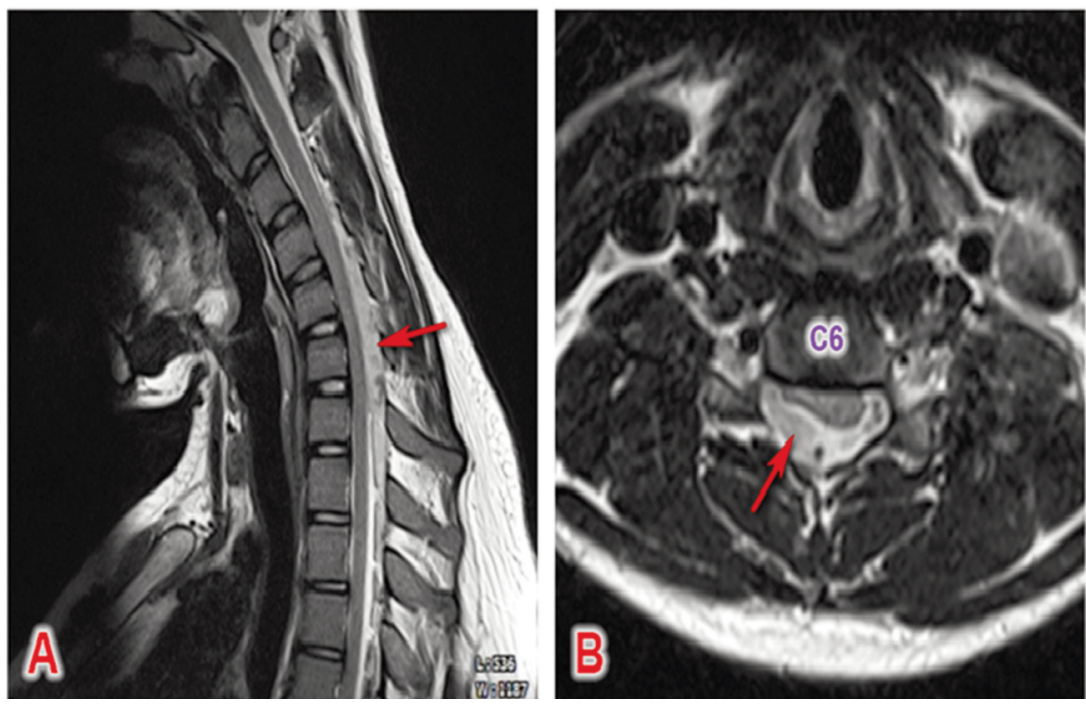

Figure 6. Cervical MRI of the 18-year-old patient in the flexion position. (A) Sagittal T2-weighted and (B) Axial T2-weighted images at C6 level showing the enlargement of the posterior epidural space due to the anterior displacement of the posterior dura causing the spinal stenosis.

characteristic sign reported by several reports $(2,8)$. The atrophy of the biceps, triceps and Delta muscles is seldomly observed. Electromyogram also shows severe or persistent denervation of the $\mathrm{C} 7$ to T1 root territory of the nerves (3).

Past studies have found a link between pathogenesis and MRI findings $(3,9)$. The most generally known anatomical theory is the asymmetric development between the dural sac and the spine. The dural sac becomes loose in the neutral position and shapes the horizontal folds. In the flexion position, there is an increase of spinal canal inducing the stretch of dural sac. The spinal cord should not be strained because the dural sac volume is matched with the expanded length of the spinal canal. In Hirayama disease, however, the dural sac length is shorter than average. The dural sac is then forced to extent in length, which causes the separation and anterior displacement of the posterior dura mater. Consequently, the vertebra body compresses the anterior boundary of the spinal cord resulting in circulatory 
disruption of the anterior horns. The study of Hirayama et al. (4) indicated the necrosis and gliosis of the anterior horns predominantly at C7 and C8 levels. The cervical MRI in neutral position and especially in the flexion position provides the evidence to support this hypothesis. In neutral position, a focal atrophy of the spinal cord at C5-C6 levels with no compression factor on sagittal T2-weighted images is a suggestive finding $(5,9)$. It is essential to observe focal hyperintensity in the anterior horns at the same levels (8). The hallmark features of the axial T2 weighted images are a smooth cervical cord and a detachment of the posterior dural wall from the bone (2). Many previous reports have recommended cervical MRI at 30 to 40 degrees of flexion when the clinical signs and supportive MRI characteristics in the neutral position indicate Hirayama disease $(2,5,10)$. This method clearly shows the anterior displacement of the posterior dural wall which causes the vertebral body to compress the lower cervical spinal cord. The enlargement of the posterior epidural space and the flow-void signal intensity of the dilated epidural veins can be observed on both axial and sagittal T2-weighted images $(2,8)$. Our clinical cases demonstrated localized medullary atrophy at levels C5-C6 with no evidence of compression on neutral position MRI images. In one instance, a discrete detachment of the posterior dural wall was observed. However, with the cervical MRI in flexion position, both patients demonstrated the usual features of Hirayama disease. Wearing a tight cervical collar produces successful outcomes in most situations (3). For increasingly progressing cases surgery should be considered (6). Anterior cervical decompression and fusion can also alleviate neurological symptoms and improve quality of life $(11,12)$.

\section{Conclusion}

For young male patients with weakness and atrophy of the distal upper extremity muscles, Hirayama disease should be considered. Furthermore, patients with the typical clinical signs and a focal atrophy of the lower cervical spinal cord on cervical MRI in neutral position should undergo cervical MRI in flexion position for early detection and care.

\section{Conflicts of Interest}

There are no conflicts of interest to declare regarding this study.

\section{Authors' Contributions}

Nguyen Minh Duc and Nguyen Duy Hung prepared, drafted, and revised the manuscript critically for important intellectual content. Each author gave the final approval of the version to be published and agreed to be accountable for all aspects of the work, ensuring that questions related to the accuracy or integrity of any part of the work are appropriately investigated and resolved.

\section{References}

1 Yoo SD, Kim HS, Yun DH, Kim DH, Chon J, Lee SA, Lee SY and Han YJ: Monomelic amyotrophy (hirayama disease) with upper motor neuron signs: a case report. Ann Rehabil Med 39(1): 122-127, 2015. PMID: 25750881. DOI: 10.5535/arm. 2015.39.1.122

2 Narayana Gowda BS, Mohan Kumar J and Basim PK: Hirayama's disease - a rare case report with review of literature. J Orthop Case Rep 3(3): 11-14, 2013. PMID: 27298910. DOI: 10.13107/jocr.2250-0685.107

3 Dejobert M, Geffray A, Delpierre C, Chassande B, Larrieu E and Magni C: Hirayama disease: three cases. Diagn Interv Imaging 94(3): 319-323, 2013. PMID: 23332131. DOI: 10.1016/ j.diii.2012.10.008

4 Hirayama K, Tomonaga M, Kitano K, Yamada T, Kojima S and Arai K: Focal cervical poliopathy causing juvenile muscular atrophy of distal upper extremity: a pathological study. J Neurol Neurosurg Psychiatry 50(3): 285-290, 1987. PMID: 3559609. DOI: $10.1136 /$ jnnp.50.3.285

5 Hirayama K, Tsubaki T, Toyokura Y and Okinaka S: Juvenile muscular atrophy of unilateral upper extremity. Neurology 13: 373-380, 1963. PMID: 13954749. DOI: 10.1212/wnl.13.5.373

6 Agundez M, Rouco I, Barcena J, Mateos B, Barredo J and Zarranz JJ: Hirayama disease: Is surgery an option? Neurologia 30(8): 502-509, 2015. PMID: 23969297. DOI: 10.1016/j.nrl. 2013.05.005

7 Yokote A, Fukuhara K, Tsugawa J and Tsuboi Y: Juvenile muscular atrophy of the proximal upper extremity as so-called proximal-type hirayama disease: Case report and review of the literature. Case Rep Neurol 11(1): 106-111, 2019. PMID: 31011327. DOI: $10.1159 / 000495606$.

8 Anuradha S and Fanai V: Hirayama Disease: A rare disease with unusual features. Case Rep Neurol Med 2016: 5839761, 2016. PMID: 28097028. DOI: 10.1155/2016/5839761

9 Kumar M, Athwal PSS, Rhandhawa S, Kahlon S and Shiv Kumar J: Hirayama's disease in a young male: A rare case report. Cureus 11(11): e6204, 2019. PMID: 31890406. DOI: 10.7759/cureus.6204

10 Hou C, Han H, Yang X, Xu X, Gao H, Fan D, Fu Y, Sun Y, and Liu B: How does the neck flexion affect the cervical MRI features of Hirayama disease? Neurol Sci 33(5): 1101-1105, 2012. PMID: 22212811. DOI: 10.1007/s10072-011-0912-x

11 Imamura $\mathrm{H}$, Matsumoto S, Hayase M, Oda Y, Kikuchi H and Takano M: [A case of Hirayama's disease successfully treated by anterior cervical decompression and fusion]. No To Shinkei 53(11): 1033-1038, 2001. PMID: 11761912.

12 Paredes I, Esteban J, Ramos A, Gonzalez P and Rivas JJ: A severe case of Hirayama disease successfully treated by anterior cervical fusion. J Neurosurg Spine 20(2): 191-195, 2014. PMID: 24286527. DOI: $10.3171 / 2013.10$.spine 13508
Received March 30, 2020

Revised April 6, 2020

Accepted April 7, 2020 